\title{
Correction to: Data Science
}

Jing He, Philip S. Yu, Yong Shi, Xingsen Li, Zhijun Xie,

Guangyan Huang, Jie Cao, and Fu Xiao

\section{Correction to: \\ J. He et al. (Eds.): Data Science, CCIS 1179, https://doi.org/10.1007/978-981-15-2810-1}

In the originally published version the affiliation of the editor Xingsen Li on page IV has been corrected. 